\title{
Quarkonia as probes of the QGP and of the initial stages of the heavy-ion collision with ALICE
}

\author{
Ingrid McKibben Lofnes ${ }^{1, *}$, for the ALICE Collaboration \\ ${ }^{1}$ University of Bergen (Norway)
}

\begin{abstract}
Studies of quarkonium production in heavy-ion collisions can be used for probing QGP properties. The suppression and regeneration of bound quarkonium states is sensitive to the medium properties. Modifications of the quarkonium polarization in $\mathrm{Pb}-\mathrm{Pb}$ collisions with respect to $\mathrm{pp}$ collisions may give further insight into the suppression and regeneration mechanisms in the QGP. Quarkonia are also sensitive to the initial stages of heavy-ion collisions, and measurements in photonuclear collisions may help constrain the nuclear gluon-distribution at low Bjorken- $x$. In this work, recent quarkonium measurements performed by ALICE in $\mathrm{Pb}-\mathrm{Pb}$ collisions at $\sqrt{s_{\mathrm{NN}}}=5.02 \mathrm{TeV}$ will be discussed. Preliminary measurements of the inclusive $\mathrm{J} / \psi R_{\mathrm{AA}}$ measured at both forward and central rapidity will be presented. The $\mathrm{J} / \psi$ polarization measured for the first time in $\mathrm{Pb}-\mathrm{Pb}$ collisions, as well as preliminary measurements of the coherent $\mathrm{J} / \psi$ photoproduction cross section, will be discussed.
\end{abstract}

The primary goal of the ALICE experiment is the study of strongly-interacting matter, known as the quark-gluon plasma (QGP), expected to be created during high-energetic heavy-ion collisions. Quarkonium production is sensitive to the medium produced in these collisions and serves as a probe of the QGP properties. Since the heavy quarks (i.e. charm and beauty) are produced in the initial hard partonic scattering, they experience the full evolution of the fireball. Bound quarkonium states immersed in a strongly interacting medium may dissociate, suppressing the quarkonium production compared to binary-scaled proton-proton (pp) collisions at the same energy. This suppression is actually a subtle interplay of several mechanisms, among them the screening of free color charges in the medium [1]. Recent theoretical developments have shown that the melting of quarkonium states is a dynamical process, where the binding of quarkonium states is weakened over time. This weakening depends on how the medium interacts with the bound states and on the time spent in the medium [2]. If the abundance of heavy quark pairs is large enough, a regeneration process may take place, where heavy quarks (re)combine either at the QGP phase boundary [3] or throughout the evolution of the fireball [4]. Recent ALICE measurements are consistent with (re)generation of charm quarks as a dominant source of the $\mathrm{J} / \psi$ production at low transverse momentum $\left(p_{\mathrm{T}}\right)[5,6]$. A modification of the quarkonium vector state polarization measured in $\mathrm{Pb}-\mathrm{Pb}$ collisions with respect to $\mathrm{pp}$ collisions can shed light on quarkonium suppression and regeneration mechanisms in the QGP. In addition, quarkonia are sensitive to the initial state of the heavy-ion collisions. Looking at photoproduction of $\mathrm{J} / \psi$ mesons in heavy-ion collisions at the LHC, one may constrain the nuclear gluon distribution at low Bjorken- $x$, in the range $x \sim 10^{-5}-10^{-2}$ [7]. The coherent photoproduction of $\mathrm{J} / \psi$

\footnotetext{
*e-mail: ingrid.mckibben.lofnes@cern.ch
} 
mesons, which takes place at very low $p_{\mathrm{T}}(\sim 60 \mathrm{MeV} / c)$, is characterized by the coherent coupling of a photon emitted by one of the nuclei while the target nucleus remains intact. Coherent photoproduction is studied in ultra-peripheral collisions, however measurements from peripheral $\mathrm{Pb}-\mathrm{Pb}$ collisions show an excess yield of $\mathrm{J} / \psi$ at very low $p_{\mathrm{T}}$, assumed to originate from coherent $\mathrm{J} / \psi$ photoproduction [8]. In these proceedings a selection of recent quarkonium measurements obtained by ALICE at both forward and central rapidity will be discussed.

The ALICE detector is capable of measuring quarkonium states down to $p_{\mathrm{T}}=0$ at both forward and central rapidity. At forward rapidity $(2.5<y<4)$ inclusive quarkonium states are measured through the dimuon channel using the muon spectrometer. The muon spectrometer includes a front absorber for filtering muons, five tracking stations, a dipole magnet with a $3 \mathrm{Tm}$ field integral, two trigger stations and an iron wall to reject punchthrough hadrons and low momentum muons. At midrapidity $(|y|<0.9)$, charmonium states are reconstructed through the dielectron channel using the Inner Tracking System (ITS) composed of six cylindrical layers of silicon detectors and the Time Projection Chamber (TPC) providing track reconstruction and particle identification via the measurement of specific energy loss, $\mathrm{d} E / \mathrm{d} x$. A more detailed description of the ALICE detectors can be found in [9].

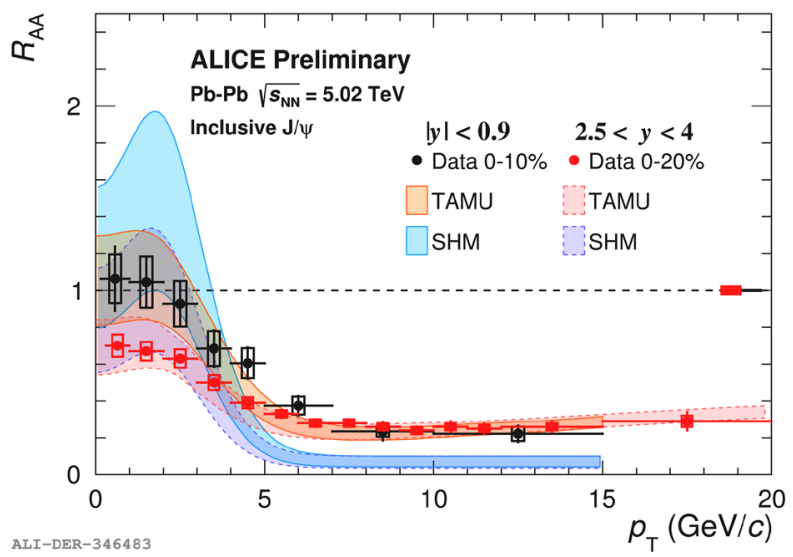

Figure 1. Preliminary measurement of the $\mathrm{J} / \psi$ nuclear modification factor in central $\mathrm{Pb}-\mathrm{Pb}$ collisions at $\sqrt{s_{\mathrm{NN}}}=5.02 \mathrm{TeV}$ at forward and central rapidity in red and black. The measurements are compared to Statistical hadronisation (SHM) and transport model (TAMU) calculations [10, 11].

The medium effect on charmonium production is quantified using the nuclear modification factor $\left(R_{\mathrm{AA}}\right)$ calculated as the ratio between the measured yield in $\mathrm{Pb}-\mathrm{Pb}$ and $\mathrm{pp}$ collisions at the same energy scaled by the number of binary collisions. Figure 1 shows the preliminary $R_{\mathrm{AA}}$ in central $\mathrm{Pb}-\mathrm{Pb}$ collisions at $\sqrt{s_{\mathrm{NN}}}=5.02 \mathrm{TeV}$, measured at both forward and central rapidity as a function of $p_{\mathrm{T}}$. These measurements are obtained from a significantly larger data sample than previous measurements at the same collisions energy, allowing for a more precise and differential result $[5,6]$. In the low $p_{\mathrm{T}}$ region $(<5 \mathrm{GeV} / c)$ the $\mathrm{J} / \psi$ mesons show less suppression at midrapidity than at forward rapidity. This is consistent with the $\mathrm{J} / \psi$ (re)generation scenario, as a higher charm cross section is expected at midrapidity. The measurements are compared to statistical hadronisation model (SHM) [10] calculations, where the production of bound states is determined by statistical weights at 
the phase boundary, and transport model (TAMU) [11] calculations assuming continuous production and dissociation of charmonium states throughout the whole QGP phase. Within the current uncertainties both models describe the observed trend.

Another physical observable is the quarkonium polarization, measured through the anisotropies in the angular distribution of the decay products,

$$
W(\cos \theta, \phi) \propto \frac{1}{3+\lambda_{\theta}} \cdot\left(1+\lambda_{\theta} \cos ^{2} \theta+\lambda_{\phi} \sin ^{2} \theta \cos 2 \phi+\lambda_{\theta \phi} \sin 2 \theta \cos \phi\right),
$$

where $\theta$ and $\phi$ are the polar and azimuthal angles in the adopted reference frame. The first measurement of $\mathrm{J} / \psi$ polarization in $\mathrm{Pb}-\mathrm{Pb}$ collisions at $\sqrt{s_{\mathrm{NN}}}=5.02 \mathrm{TeV}$ as a function of $p_{\mathrm{T}}$ was recently reported by ALICE [12], showing a $\lambda_{\theta}$ with a maximum of $2 \sigma$ deviation with respect to zero in the $p_{\mathrm{T}}$ interval from $2-4 \mathrm{GeV} / c$. The preliminary measurement of the $\mathrm{J} / \psi$ polarization at forward rapidity in $\mathrm{Pb}-\mathrm{Pb}$ collisions at $\sqrt{s_{\mathrm{NN}}}=5.02 \mathrm{TeV}$ as a function of centrality is shown in Fig. 2 for $2<p_{\mathrm{T}}<6 \mathrm{GeV} / c$. The polarization parameters show a flat trend in both the helicity and Collins-Soper reference frames. A non-zero $\lambda_{\theta}$ is observed in both frames, with slightly positive values in the helicity frame and slightly negative values in the Collins-Soper frame.

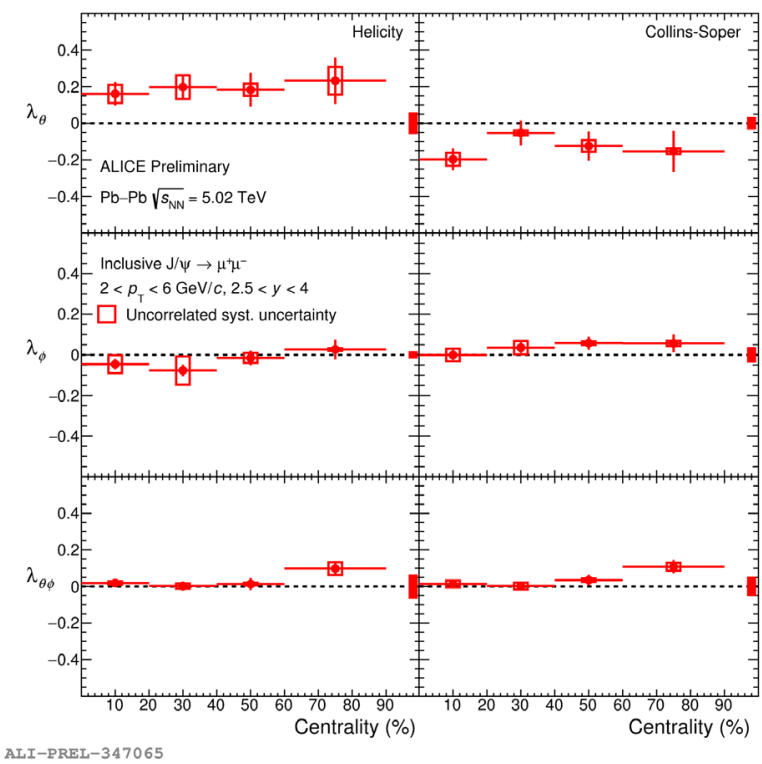

Figure 2. $\mathrm{J} / \psi$ polarization parameters as a function of centrality measured at forward rapidity in $\mathrm{Pb}-\mathrm{Pb}$ collisions at $\sqrt{s_{\mathrm{NN}}}=5.02 \mathrm{TeV}$.

The preliminary measurement of the coherent $\mathrm{J} / \psi$ photoproduction cross section at $\sqrt{s_{\mathrm{NN}}}=5.02 \mathrm{TeV}$ measured at forward rapidity is shown in Fig. 3. The measured coherent $\mathrm{J} / \psi$ photoproduction cross section shows an increase by a factor $\sim 3$ with respect to the coherently photoproduced $\mathrm{J} / \psi$ at $\sqrt{s_{\mathrm{NN}}}=2.76 \mathrm{TeV}$ [8]. The coherent photoproduction cross section is compared with models implementing a modification of the photon flux with respect to ultra-peripheral collisions (UPC). A light cone color dipole formalism was used 


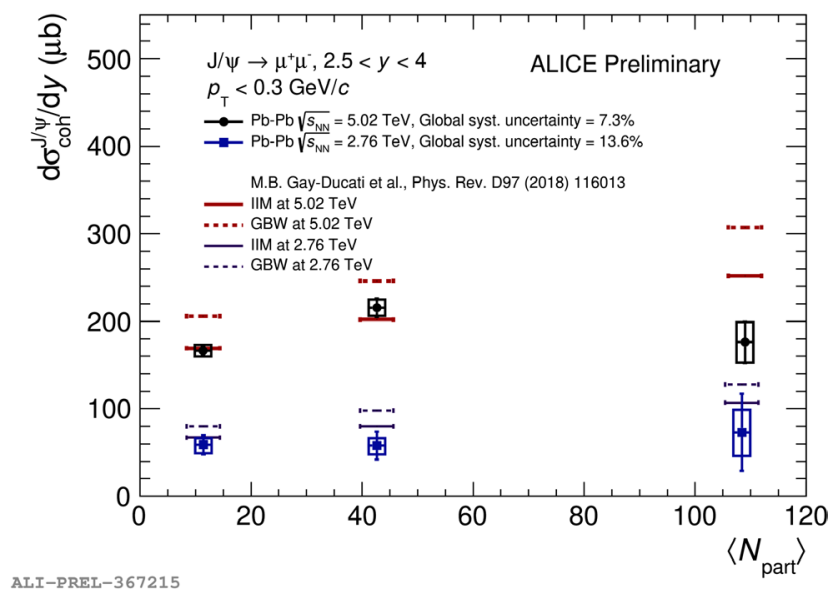

Figure 3. $\mathrm{J} / \psi$ coherent photoproduction cross section measured at forward rapidity in $\mathrm{Pb}-\mathrm{Pb}$ collisions at $\sqrt{s_{\mathrm{NN}}}=5$ and $2.76 \mathrm{TeV}[8]$ in black and blue, respectively.

in the Golec-Biernat-Wusthoff (GBW) calculation, while the Iancu-Itakura-Munier (IIM) calculation is based on the Color Glass Condensate approach [13]. A qualitative agreement is observed for the most peripheral collisions, while the measurements in semicentral events deviate from the model predictions.

In summary, ALICE provides a comprehensive set of quarkonium results in heavy-ion collisions. The inclusive $\mathrm{J} / \psi R_{\mathrm{AA}}$ measured at both forward and central rapidity as a function of transverse momentum is consistent with a significant contribution from (re)generation in the low $p_{\mathrm{T}}$ region. The $\mathrm{J} / \psi$ polarization is measured for the first time in $\mathrm{Pb}-\mathrm{Pb}$ collisions, showing a flat trend for all polarization parameters as a function of centrality, with a non-zero $\lambda_{\theta}$. Measurements of the coherent $\mathrm{J} / \psi$ photoproduction cross section at forward rapidity show a considerable increase in the cross section for increasing center-of-mass energy. The photoproduction cross section is well described by UPC-based models in peripheral collisions, but deviations are observed for semicentral collisions.

\section{References}

[1] T. Matsui and H. Satz, Phys. Lett. B 178 416-422 (1986)

[2] A. Rothkopf, Phys. Rept. 858 1-117 (2020)

[3] P. Braun-Munzinger and J. Stachel, Phys. Lett. B 490 196-202 (2000)

[4] R. L. Thews, M. Schroedter, and J. Rafelski, Phys. Rev. C 63, 054905 (2001)

[5] ALICE Collaboration, S. Acharya et al., Phys. Lett. B 805135434 (2020)

[6] ALICE Collaboration, S. Acharya et al., JHEP 02041 (2020)

[7] J. G. Contreras, Phys. Rev. C 96015203 (2017)

[8] ALICE Collaboration, J. Adam et al., Phys. Rev. Lett. 116222301 (2016)

[9] ALICE Collaboration, K. Aamodt et al., JINST 3 S08002 (2008)

[10] A. Andronic et al., Phys. Lett. B 979134836 (2019)

[11] X. Du and R. Rapp, Nucl. Phys. A 943 147-158 (2015)

[12] ALICE Collaboration, S. Acharya et al., Phys. Lett. B 815136146 (2021)

[13] M. G. Ducati and S. Martins, Phys. Rev. D 97116013 (2018) 